\title{
IL-17C promotes immune-mediated glomerular disease
}

The IL-17 family of cytokines are produced by Thelper $17\left(\mathrm{~T}_{\mathrm{H}} 17\right)$ cells and have an important role in organ-specific autoimmunity. As most of the studies of this role to date have focused on IL-17A, the specific functions of the other IL-17 cytokines are less well understood. Now, Ulf Panzer and colleagues report a pathogenic role of IL-17C-IL-17 receptor E (IL-17RE) signalling in immune-mediated glomerular disease.

The researchers found that serum levels of IL-17C, but not of IL-17A, IL-17E or IL-17F, were significantly increased in patients with acute anti-neutrophil cytoplasmic antibody-associated crescentic glomerulonephritis compared with healthy individuals. Expression of IL-17C and its specific receptor IL-17RE were also upregulated in a mouse model of crescentic glomerulonephritis. In this model, deficiency of IL-17C or IL-17RE had a protective effect, resulting in a reduction in glomerular crescent formation and tubulointerstitial injury as well as less severe kidney dysfunction compared with wild-type mice. Similarly, IL-17C deficiency reduced disease severity in a mouse model of lupus nephritis.

"Mechanistically, we provide evidence that the IL-17C-IL-17RE axis directly stimulates the nephritogenic $T_{H} 17$ cell response and thereby promotes renal tissue injury in crescentic glomerulonephritis," says Panzer. "Based on our finding that IL-17C stimulates the $T_{H} 17$ cell response upstream of IL-17A, targeting of the IL-17C-IL-17RE pathway may present an intriguing therapeutic strategy for $\mathrm{T}_{\mathrm{H}} 17$-driven autoimmune and inflammatory diseases." The researchers are now investigating the therapeutic potential of a neutralizing IL-17C antibody in crescentic glomerulonephritis.

Ellen F. Carney

ORIGINAL ARTICLE Krohn, S. et al. IL-17C/IL-17 receptor E signaling in $\mathrm{CD} 4^{+} \mathrm{T}$ cells promotes $\mathrm{T}_{\mathrm{H}} 17$ cell-driven glomerular inflammation.J.Am. Soc. Nephrol. https://doi.org/10.1681/ ASN.2017090949 (2018)

FURTHER READING Krebs, C. F. et al. Thelper type 17 cells in immune-mediated glomerular disease. Nat. Rev. Nephrol. 13, $647-659$ (2017)

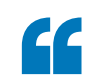

the IL-17CIL-17RE axis directly stimulates the nephritogenic $\mathrm{T}_{\mathrm{H}} 17$ cell response and thereby promotes renal tissue injury 